\title{
Incentive Contracting Under Ambiguity Aversion
}

Liu, Qi, Lei Lu, and Bo Sun

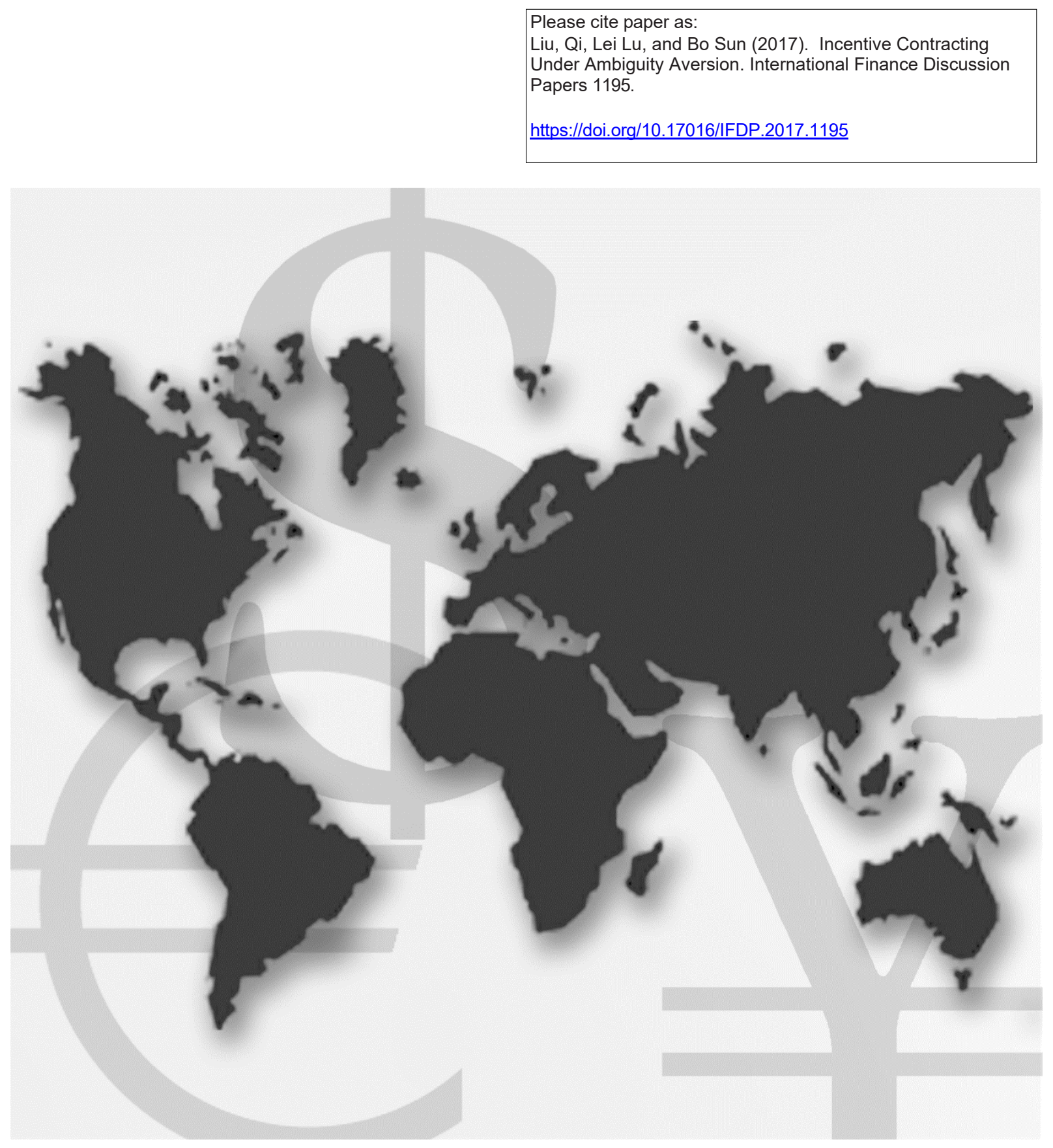

\section{International Finance Discussion Papers}

Board of Governors of the Federal Reserve System

Number 1195

February 2017 
Board of Governors of the Federal Reserve System

International Finance Discussion Papers

Number 1195

February 2017

\section{Incentive Contracting Under Ambiguity Aversion}

Qi Liu, Lei Lu, Bo Sun

NOTE: International Finance Discussion Papers are preliminary materials circulated to stimulate discussion and critical comment. References to International Finance Discussion Papers (other than an acknowledgment that the writer has had access to unpublished material) should be cleared with the author or authors. Recent IFDPs are available on the Web at www.federalreserve.gov/pubs/ifdp/. This paper can be downloaded without charge from the Social Science Research Network electronic library at www.ssrn.com. 


\title{
Incentive Contracting Under Ambiguity Aversion*
}

\author{
Qi Liu \\ Peking University \\ Lei Lu \\ University of Manitoba \\ Bo Sun \\ Federal Reserve Board
}

\begin{abstract}
This paper studies a principal-agent model in which the information on future firm performance is ambiguous and the agent is averse to ambiguity. We show that if firm risk is ambiguous, while stocks always induce the agent to perceive a high risk, options can induce him to perceive a low risk. As a result, options can be less costly in incentivizing the agent than stocks in the presence of ambiguity. In addition, we show that providing the agent with more incentives would induce the agent to perceive a higher risk, and there is a discontinuous jump in the compensation cost as incentives increase, which makes the principal reluctant to reset contracts frequently when underlying fundamentals change. Thus, compensation contracts exhibit an inertia property. Lastly, the model sheds some light on the use of relative performance evaluation, and provides a rationale for the puzzle of pay-for-luck in the presence of ambiguity.
\end{abstract}

Keywords: Ambiguity, Executive compensation, Options, Relative performance evaluation

JEL Classification: G30, J33

*We thank Nicholas Yannelis (the Editor) and two anonymous referees for helpful comments. The views expressed herein are the authors' and do not necessarily reflect the opinions of the Board of Governors of the Federal Reserve System. Qi Liu acknowledges support by National Natural Science Foundation of China (No. 71502004). 


\section{Introduction}

The vast and growing literature on the principal-agent relationship with moral hazard is usually based on the assumption that both contracting parties are expected utility maximizers. In particular, there is only a single prior belief of the distribution of future firm performances. However, lack of information in start-up companies and emerging industries may cause individual knowledge about future firm performances to be ambiguous, that is, multiple possible distributions exist.

We study a principal-agent model in which there are multiple possible probability distributions of future performances, and the agent is both risk-averse and ambiguity-averse. The feature of ambiguity-aversion is consistent with the mounting experimental evidence, including the most notable example Ellsberg Paradox (Ellsberg (1961)) and portfolio choice experiments (Ahn, Choi, Gale, and Kariv (2009)). Gilboa and Schmeidler (1989) provide an axiomatic basis for a "max-min" preference to model ambiguity-aversion. Following the literature, we use the "max-min" preference to model the agent's behavior. In particular, if firm risk is ambiguous, the agent's effort and utility are determined in the following two steps: first, given the agent's effort, the agent perceives a level of risk that minimizes his utility; second, the agent chooses an effort level to maximize the utility that has been minimized under possible risks.

Our analysis contributes to the compensation literature in that we provide a new, coherent theoretical explanation for a number of empirical findings that have not been consistently reconciled with the existing theory, namely, the optimality of option grants, an inertia property of compensation contracts, and the varied use of relative performance 
evaluation. We discuss each of them in turn.

First, we show that if there is ambiguity on firm risk, while stocks always induce the agent to perceive the highest possible risk, options can induce him to perceive the lowest possible risk. As a result, options can be less costly than stocks in incentivizing the agent in the presence of ambiguity. In other words, the use of options will induce the agent to underestimate risk, leading to overconfidence on the part of managers. While the literature on overconfident managers takes under-estimation of risk as assumptions, this paper derives them as a result for an ambiguity-averse agent.

We illustrate the intuition as below: when firm risk is ambiguous, an increase in the firm risk only increases the variance of stocks, but has no effects on the mean value of stocks. A risk-averse and ambiguity-averse agent will always perceive the highest risk when he owns stocks. Therefore, a high risk premium in compensation is required. On the other hand, an increase in firm risk increases both the mean payoff and variance of options. While an increase in the variance of options decreases the agent's utility, an increase in the mean payoff of options also has a positive effect on his utility. If the agent is not too risk-averse, he could perceive a low risk. As a result, only a low risk premium is required. If the reduction in risk premium is sufficiently large, options can be less costly than stocks in providing appropriate incentives.

Our analysis thus provides a new rationale for the popularity of option compensation that has triggered much debate in the literature. The calibration of a standard principalagent model shows that options should not be a part of an optimal contract (Dittmann and Maug (2007)), rendering it puzzling as to why options are widely used in practice. 
While risk-taking incentives have been proposed as the cause (Feltham and Wu (2001), Dittmann and $\mathrm{Yu}(2011)){ }^{1}$, several papers show that it is not always true that giving options to agents will make them more willing to take risk (e.g. Carpenter (2000), Ross (2004)). Bettis, Bizjak, and Lemmon (2005) also show that managers exercise their options earlier when volatility increases, and Hayes, Lemmon and Qiu (2010) find little evidence to support the conventional wisdom that the convexity inherent in option compensation is used to reduce risk-related agency problems. Our model shows that options can be a cost-effective compensation vehicle in the presence of ambiguous payoff distributions.

Second, we show that compensation contracts may be irresponsive to changes in fundamentals, offering a new explanation for the empirical pattern that compensation contracts are rather rigid (Bizjak, Lemmon, and Naveen (2008), Faulkender and Yang (2010)). In particular, our analysis indicates that providing the agent with more incentives would induce the agent to perceive a higher risk; When the target effort goes beyond a threshold, the agent's perception of risk will jump from the lowest risk to the highest one. This also implies a discontinuous jump in the risk premium paid to the agent. Given the discontinuous jump in the compensation cost, the principal will be reluctant to reset contracts frequently when underlying fundamentals change. Compensation contracts, therefore, exhibit an inertia property.

Lastly, when the correlation between observable shocks and unobservable shocks is ambiguous, we find that while using relative performance evaluation (hereafter, RPE) to filter out these observable returns from the agent's payoff reduces the cost of stock compensation, it can increase the cost of option compensation (because options induce

\footnotetext{
${ }^{1}$ Dittmann, Maug and Spalt (2010) justify the use of options based on loss-averse managers.
} 
the agent to perceive a negative correlation between observable shocks and unobservable shocks, and thus a low total firm risk). The model thus predicts that RPE should be more frequently used for stock compensation than option compensation. Gong, Li and Shin (2010) and Albuquerque (2012) find that RPE is less likely to be used if firms have greater growth opportunities. De Angelis and Grinstein (2011) find that firms rely less on RPE when they are smaller or belong to high-growth industries. To the extent that firms with smaller sizes or greater growth opportunities are associated with more option compensation in managerial pay (as it allows these companies to conserve current cash flow while providing significant potential upside incentives; see empirical evidence in, for example, Smith and Watts (1992) and Gaver and Gaver (1993)), the less frequent use of RPE in these firms can be consistent with our theory. Moreover, we show that conditions exist under which non-indexed options are less costly than indexed stock to induce effort. This result also sheds some light on the pay-for-luck puzzle in the compensation literature (Bertrand and Mullainathan (2001)).

This paper builds on the literature on agency problem with ambiguity-averse agents. Karni (2009) develops axiomatic foundations of ambiguity aversion in a principal-agent model. Weinschenk (2010) considers linear contracts and shows that the principal may optimally provide no incentives for a subset of informative performance measures in the presence of ambiguity. ${ }^{2}$ Mukerji (2010) studies the impact of ambiguity in procurement contracts under cost uncertainty, and finds that the optimal pay for performance decreases with both the ambiguity and the ambiguity aversion of the agent relative to that of the

\footnotetext{
${ }^{2}$ Since Weinschenk (2010) only considers linear contracts, the paper shows that the ambiguity-averse agent is always induced to perceive the lowest possible mean value of the shock. If the shock has a sufficiently low possible mean value, the principal may choose not to contract on such a performance measure due to the participation constraint.
} 
principal. Viero (2012) shows that the optimal contract can have distortion of effort even when the agent is risk neutral, through motives to bet on the resolution of information vagueness. Szydlowski (2012) shows that the agent receives excessively strong incentives in a dynamic contracting setting when the principal is ambiguous about the agent's cost of effort. Kellner (2015) studies a principal-agent problem with multiple agents and shows that ambiguity and ambiguity aversion can lead to the superiority of tournaments over wage schemes that only depend on each agent's own output level. ${ }^{3}$. A recent paper by Carroll (2015) derives the optimality of linear contracts under the assumption that the principal is uncertain what the agent can and cannot do. If the principal evaluates possible contracts by their worst-case performance over unknown actions the agent might potentially take, then the optimal contract is linear. Differen from the literature, our paper focuses on comparing linear contracts (stock compensation) with non-linear contracts (option compensation) in the presence of ambiguity. ${ }^{4}$ In particular, we show that option compensation can be less costly, since it can induce the ambiguity-averse agent to perceive a low risk.

The remainder of the paper is organized as follows. Section 2 sets up the model and contrasts it with a model without ambiguity. Section 3 discusses the contracting results when there is ambiguity on firm risk. Section 4 discusses the robustness of results. Section 5 concludes. All proofs are contained in the Appendix.

\footnotetext{
${ }^{3}$ Kellner and Riener (2012) provide experimental evidence for Kellner (2015)

${ }^{4}$ Our paper is also related to studies on principal-agent problems with uncertainty. For example, $\mathrm{He}$, Li, Wei and $\mathrm{Yu}$ (2014) prove a positive relation between uncertainty and incentives with ambiguityneutral agents, which helps reconcile mixed empirical evidence on the relation between risk and incentives. Standard principal-agent models predict a negative relation between risk and incentives. But empirical evidence is mixed. While some papers find a negative relation, others document no significant or even a positive relation. For a review of the literature, see Prendergast (2002).
} 


\section{Model}

\subsection{The benchmark model without ambiguity}

Consider a one-period principal-agent model: the principal (she) hires an agent (he) to run the firm. At time $t=0$, after the contract is offered, the agent exerts an effort, denoted by $a$, which is unobservable to the principal. we assume that a feasible set of effort lies in a bounded interval, i.e. $a \in[\underline{a}, \bar{a}]$. The upper bound $\bar{a}$ reflects the fact that there is a limit on the number of positive net-present-value (NPV) projects that the agent can undertake or a limit on the number of working hours that the agent can spend. At time $t=1$, firm value is given by $x=a+l+\epsilon$, where $\epsilon$ is a noise term, which is normally distributed with mean zero and variance $\sigma^{2}$. The noise is realized after the agent exerts effort, and $l$ is a constant term, which can be interpreted as firms' base value or expected market returns that are beyond the agent's control.

The mean and variance of an option with an exercise price $k$ are given by

$$
\begin{aligned}
m_{k} & =\sigma[\phi-(1-\Phi) \eta] \\
\sigma_{k}^{2} & =\sigma^{2}\left[\left(1-\Phi-\phi^{2}\right)-\phi(2 \Phi-1) \eta+\Phi(1-\Phi) \eta^{2}\right]
\end{aligned}
$$

where

$$
\begin{aligned}
\eta & =\frac{k-a-l}{\sigma} \\
\phi & =\frac{1}{\sqrt{2 \pi}} \exp \left(-\frac{1}{2} \eta^{2}\right) \\
\Phi & =\int_{-\infty}^{\eta} \frac{1}{\sqrt{2 \pi}} \exp \left(-\frac{1}{2} t^{2}\right) d t
\end{aligned}
$$


Similar to Feltham and $\mathrm{Wu}$ (2001), we formally state the properties of the mean and variance of an option with an exercise price $k$ below.

Lemma 1. The mean value and variance of an option with an exercise price $k$ (i.e., $m_{k}$ and $\sigma_{k}^{2}$ respectively) are increasing in $a, l$ and $\sigma$, and decreasing in $k$.

Since the agent's action is unobservable, the principal can only contingent the agent's pay on the realized firm value. For tractability, we restrict contracts to be either stock compensation (fixed payment + stocks) or option compensation (fixed payment + options with an exercise price $k$ ). An option compensation consists of a fixed payment $\alpha_{k}$ and $\beta_{k}$ shares of options with the exercise price $k$. The agent's payoff with option compensation is $w=\alpha_{k}+\beta_{k} \max (x-k, 0)$. For stock compensation with a fixed payment $\alpha_{s}$ and $\beta_{s}$ shares of stocks, we consider it as a linear contract, and the payoff is represented by $w=\alpha_{s}+\beta_{s} x$.

The agent is risk-averse and his expected utility is given by the following mean-variance preference: ${ }^{5}$

$$
E[u]=E[w]-\frac{1}{2} \lambda \operatorname{Var}[w]-g(a)
$$

where $g(a)=\frac{1}{2} a^{2}$ is the cost of exerting an effort $a$, and $\lambda$ represents the agent's riskaversion.

The principal is risk-neutral. Her objective is to maximize the expected firm value net of the agent's pay: ${ }^{6}$

$$
\max _{w} E[x-w]
$$

\footnotetext{
${ }^{5}$ Here we follow Feltham and $\mathrm{Wu}(2001)$ to use mean-variance preference for tractability. The meanvariance preference is usually a proxy for a standard expected utility (it is equivalent to the expected exponential utility when the contract is linear), and captures the key feature of a general utility function that the agent likes a high payoff, but dislikes the variance of a payoff. In Section 4, we provide a robustness check to show that the key mechanism for our main results still holds for a general utility function.

${ }^{6}$ Here we normalize the reservation utility to be 0 .
} 
subject to the individual rationality constraint (IR): $E[u(a)] \geq 0$, and the incentive compatibility constraint (IC): $a \in \arg \max _{\hat{a}} E[u(\hat{a})]$.

In order to get closed-form solutions, we fix the target effort $a$, and study the optimal way (stock versus options) to induce the effort. When the target effort is fixed, the principal's objective is equivalent to minimizing the cost of the agent's contract. In the paper, we will compare the cost of stock compensation, denoted by $C_{s}$, with the cost of option compensation, denoted by $C_{k}$, to implement the effort, and provide a graphical illustration in Figure 1. The results are similar to those in Hall and Murphy (2000) and Feltham and $\mathrm{Wu}(2001)$.

Proposition 1. Denote $C_{s}$ as the cost of stock compensation to implement the target effort $a$, and $C_{k}$ as the cost of option compensation to implement a. For any $k>0, C_{s}<C_{k}$.

There are two complementary perspectives on the choice between stock and option compensation. One perspective highlights that options are more "expensive" since risk-averse executives value options at significantly less than the cost to the company (e.g., Hall and Murphy (2000)). The economic or opportunity cost of granting an option is the amount the company could have received if it were to sell the option to an outside investor, who is generally considered risk-neutral, free to trade the option and take actions to hedge away the risk of the option. In contrast to outside investors, company executives cannot trade their options and are forbidden from hedging the risks by short-selling company stock. In addition, while outside investors tend to be well-diversified, company executives are inherently undiversified, with their physical as well as human capital invested disproportionately in their company. For these reasons, company executives will generally place a much lower 
value on company stock options than would outside investors. The other perspective emphasizes that options provide more incentives for the same dollar outlay as an equivalent investment in stocks. If the base salary is rigid, the cost of stock compensation or option compensation is proportional to market value of stocks or options. In this case, options are less costly in providing identical incentives. This is illustrated in the top graph of Figure 1, which shows that market value is decreasing in the exercise price. However, if the base salary is adjustable, since stocks provide more subjective values to the agent than options, stock compensation can result in a lower base salary necessary compared to option compensation. Then it can be shown that the cost of a contract is proportional to the risk premium paid to the agent. As a result, stocks dominate options as shown in Proposition 1 and the bottom graph of Figure 1. The intuition here is also illustrated in Dittmann and Maug (2007). Below we will show that options can be a desirable compensation instrument in the presence of ambiguity.

\subsection{A model with ambiguity aversion}

In the benchmark model without ambiguity, the firm value $x$ is normally distributed with mean $a+l$ and variance $\sigma^{2}$. The agent infers his expected utility from this objective distribution. But in start-up companies and emerging industries, lack of reliable information could cause the agent to face ambiguity regarding risk and returns. In this section, we study the contracting implications when firm risk is ambiguous, that is, the value of $\sigma$ lies in a closed interval $\left[\sigma_{1}, \sigma_{2}\right], \sigma_{1} \leq \sigma_{2}$.

Following the literature on ambiguity-aversion, we assume that the agent is both risk- 
averse and ambiguity-averse. When faced with ambiguity, given any level of effort, he first minimizes his utility under possible distributions (i.e., his utility is inferred in the worst case), and then choose an effort to maximize his utility that has been minimized under possible distributions. The agent's objective is thus given by

$$
\max _{\hat{a}} \min _{\sigma \in\left[\sigma_{1}, \sigma_{2}\right]} E[u(\hat{a})]
$$

Note that when the agent exerts different levels of effort, the distributions of firm value will be changed, and thus, his perception of distributions (i.e., the worst distribution for the agent) can be different.

There is no asymmetric information between the principal and the agent regarding the range of risk, that is, the principal's perception of risk, denoted by $\sigma_{p}$, is $\sigma_{p} \in\left[\sigma_{1}, \sigma_{2}\right]$. Since the principal can diversify her risk in the market, she may be less averse to ambiguity on risk. Here we do not make any assumptions on the principal's attitude towards ambiguity, ${ }^{7}$ and the key driving forces underlying our results is that the agent is ambiguity-averse. Given the target effort $a$, the principal's objective is to minimize the cost of implementing a. Since the effort cost is always $\frac{1}{2} a^{2}$, we drop this constant term in the rest of the paper. The cost below refers to the risk premium paid to the agent.

\footnotetext{
${ }^{7}$ It can be shown that if the principal is ambiguity-averse, $\sigma_{p}=\sigma_{2}$.
} 


\section{Results}

\subsection{Optimality of options}

Lemma 2. Let $\sigma_{a}$ and $\sigma_{p}$ be the agent's and the principal's perception of risk, respectively.

1) If the principal uses stock compensation to induce effort, then the agent's perception of risk is $\sigma_{a}=\sigma_{2}$; the cost of stock compensation is $C_{s}=\frac{1}{2} \lambda a^{2} \sigma_{2}^{2}$.

2) If the principal uses option compensation to induce effort, let $\beta_{k}\left(\sigma_{1}\right)$ be the number of options required to induce the target effort a if the agent's perception of risk is $\sigma_{1}$, i.e., $a \in \arg \max _{\hat{a}} E\left[u(\hat{a}) \mid \sigma_{a}=\sigma_{1}\right.$ and $\left.\beta_{k}=\beta_{k}\left(\sigma_{1}\right)\right]$. If the agent is granted $\beta_{k}\left(\sigma_{1}\right)$ options with an exercise price $k$, and

$$
\lambda<\min _{\sigma \in\left[\sigma_{1}, \sigma_{2}\right]} \frac{2}{\beta_{k}\left(\sigma_{1}\right)} \frac{m_{k}(\sigma)-m_{k}\left(\sigma_{1}\right)}{\sigma_{k}^{2}(\sigma)-\sigma_{k}^{2}\left(\sigma_{1}\right)}
$$

then the agent will exert the effort a, his perception of risk is $\sigma_{1}$ and the cost of option compensation is $C_{k}=\frac{1}{2} \lambda \beta_{k}^{2}\left(\sigma_{1}\right) \sigma_{k}^{2}\left(\sigma_{1}\right)+\beta_{k}\left(\sigma_{1}\right)\left[m_{k}\left(\sigma_{p}\right)-m_{k}\left(\sigma_{1}\right)\right]$.

The intuition behind Lemma 2 is illustrated as follows. The mean and variance of stocks are given by $a+l$ and $\sigma^{2}$. An increase in $\sigma$ has no effects on the mean value of stocks, but increases the variance of stock. The risk-averse agent will always perceive the highest risk $\sigma_{2}$ when he owns stocks. However, for options, an increase in $\sigma$ increases both the mean value and variance of options. While an increase in the variance of options decreases the agent's utility, an increase in the mean value of options also has a positive effect on his utility. Thus whether the agent perceives a high risk or low risk depends on which effect dominates. If the agent is not too risk-averse, the effect from an increased mean value of 
options is dominant, and thus the agent's utility is minimized at a low risk. Therefore, the agent will perceive a low risk.

In the presence of ambiguity, the cost of option compensation can be decomposed into two parts: the risk premium paid to the agent and the wedge in the valuation of options between the principal and the agent (because now their perceptions of risk may be different). Since the mean value of stocks is not affected by risk, there is no difference in the valuation of stock between the principal and the agent. Therefore, the cost of stock compensation involves only the risk premium required. Stocks always induce the agent to perceive a high risk. As a result, the principal has to pay a high risk premium. However, as argued above, options can induce the agent to perceive a low risk. The perception of a low risk has two counteracting effects on the cost of option compensation. On the one hand, it reduces the risk premium; on the other hand, it increases the valuation wedge of options between the principal and the agent. If the agent's risk aversion is large enough, the first effect will dominate, so that the cost of option compensation decreases. The following proposition shows that options are less costly than stocks for intermediate values of the risk aversion on the part of the agent $(\lambda)$.

Proposition 2. Suppose $\sigma_{1}$ is small enough such that $a^{2} \sigma_{2}^{2}>\beta_{k}^{2}\left(\sigma_{1}\right) \sigma_{k}^{2}\left(\sigma_{1}\right)$. If the agent's risk aversion $\lambda$ satisfies

$$
\frac{2 \beta_{k}\left(\sigma_{1}\right)\left[m_{k}\left(\sigma_{p}\right)-m_{k}\left(\sigma_{1}\right)\right]}{a^{2} \sigma_{2}^{2}-\beta_{k}^{2}\left(\sigma_{1}\right) \sigma_{k}^{2}\left(\sigma_{1}\right)}<\lambda<\min _{\sigma \in\left[\sigma_{1}, \sigma_{2}\right]} \frac{2}{\beta_{k}\left(\sigma_{1}\right)} \frac{m_{k}(\sigma)-m_{k}\left(\sigma_{1}\right)}{\sigma_{k}^{2}(\sigma)-\sigma_{k}^{2}\left(\sigma_{1}\right)}
$$

then it is less costly to use options (with an exercise price $k$ ) than stocks to induce effort.

The right-hand side of the inequality ensures that the agent's perception of risk is $\sigma_{1}$ 
with options (by Lemma 2). Suppose $\beta_{k}\left(\sigma_{1}\right)$ is larger, i.e., the number of options granted to the agent to induce the target effort is larger. Since the agent is risk-averse and ambiguityaverse, it will become more difficult to induce him to perceive a low risk. The upper bound for $\lambda$ has to be consequently decrease to ensure that the agent can be induced to perceive a low risk with options. The other term in the right-hand side, $\min _{\sigma \in\left[\sigma_{1}, \sigma_{2}\right]} \frac{m_{k}(\sigma)-m_{k}\left(\sigma_{1}\right)}{\sigma_{k}^{2}(\sigma)-\sigma_{k}^{2}\left(\sigma_{1}\right)}$, measures the increment of the mean value of options relative to the increment of the variance of options, when firm risk goes up. As this term becomes higher, the increase in firm risk has a larger effect on the mean value of options, implying that it will be easier to induce the agent to perceive a low risk with options. The upper bound for $\lambda$ increases as a result.

The left-hand side of the inequality ensures that it is less costly to use options than stocks to induce effort (by comparing the cost of both contracts from Lemma 2). If $\beta_{k}\left(\sigma_{1}\right)$ is higher, i.e., the principal needs to use more options to induce the target effort, option compensation consequently becomes more costly. requiring the lower bound for $\lambda$ to increase. If $\sigma_{p}$ is larger, which means that the principal recognizes a higher risk, and thus, a higher value to options, then options in managerial compensation become less appealing. Therefore, the lower bound for $\lambda$ increases. Lastly, if $\sigma_{2}$ becomes larger, a higher risk premium is required for stock compensation, making option compensation more appealing and the lower bound for $\lambda$ decrease. The following numerical example shows that the condition (6) can be satisfied.

Example 1: In the condition (6), $\beta_{k}\left(\sigma_{1}\right)$ depends on $\lambda$, so it is not clear whether this condition can be satisfied in a reasonable range of $\lambda$. In the Appendix, we show that the 
condition (6) can be translated into a lower and upper bound of $\lambda$. In Figure 2, we provide a numerical example to show that the condition (6) can be actually satisfied in a reasonable range of $\lambda$.

In the example, we set $a=0.5, k=0.4, l=0, \sigma_{1}=0.1$, and $\sigma_{2}=0.5$. From Figure 2 , we can see that as the shareholders' perception of risk $\sigma_{p}$ goes from $\sigma_{1}$ to $\sigma_{2}$, the upper bound of $\lambda$ is not affected, and equals to a constant 4.66. This is expected, since the righthand side of (6) is not affected by $\sigma_{p}$. As $\sigma_{p}$ goes from $\sigma_{1}$ to $\sigma_{2}$, the lower bound increases from 0 to 3 . This is also consistent with the fact that the left-hand side of (6) increases in $\sigma_{p}$. In sum, as $\sigma_{p}$ goes from $\sigma_{1}$ to $\sigma_{2}$, the range for $\lambda$ that satisfies the condition (6) changes from $[0,4.66]$ to $[3,4.66]$.

\subsection{Inertia property of compensation contracts}

In this subsection, we study the conditions under which the agent will perceive a low risk. In reality, both stock and options are widely used in compensation. In an effort to be consistent with the reality, we allow contracts to contain both stock and options in this

part. Specifically, we assume that a contract consists of a fixed payment $\alpha, \beta_{s}$ shares of stock, and $\beta_{k}$ options of exercise price $k$. The payoff of the contract is represented by

$$
w=\alpha+\beta_{s} x+\beta_{k} \max (x-k, 0) .
$$

Intuitively, the amount of incentives will affect whether the agent perceives a high or low risk. Because the agent is risk-averse, he is more likely to perceive a higher risk if he is granted more incentives. When the target effort rises, higher powered compensation is 
required to induce effort. As a result, the agent tends to perceive a high risk.

Lemma 3. Given two levels of effort $a_{1}<a_{2}$, if $a_{2}$ is implemented and the agent perceives $\sigma_{1}$, then $a_{1}$ can also be implemented while keeping the agent's perception of risk unchanged.

Lemma 3 shows that it becomes more difficult to induce the agent to perceive $\sigma_{1}$, as the target effort increases. The next lemma will show that under some conditions, the agent's perception of risk will quickly jump from $\sigma_{1}$ to $\sigma_{2}$. Given a contract, the agent's utility is jointly determined by the expected value and the variance of his compensation payments. The expected value and variance both increase in the agent's perception of risk. Note that the expected value is proportional to the level of incentives, and the variance is proportional to the variability in the level of incentives. If risk is not too small and incentives are weak, the expected value dominates in determining the agent's perception of risk, and therefore the agent will perceive the lowest risk $\sigma_{1}$; with strong incentives, however, the variance component is a dominant force, and the agent will perceive the highest risk $\sigma_{2}$.

Lemma 4. Suppose that the target effort a is implemented. If

$$
|k-a-l|<\sigma_{1} \cdot \min \left(1, \sqrt{\frac{1}{2} \lambda a \sigma_{1}}\right),
$$

then the agent's perception of risk is either the lowest risk $\sigma_{1}$ or the highest risk $\sigma_{2}$.

Lemma 3 and 4 imply that there exists a cut-off effort level, denoted by $a_{0}$, such that for any effort level below $a_{0}$, the principal can implement effort with a contract that enables the agent to perceive the lowest risk. In this case, a low risk premium is required. However,

\footnotetext{
${ }^{8}$ In reality, firms usually use at-the-money options to compensate their managers. Then $k$ is roughly equal to $E[x]=a+l$. The condition (7) is satisfied automatically.
} 
for any effort level above $a_{0}$, incentives required to induce effort are sufficiently strong that the agent will perceive the highest risk. In this case, a high risk premium is required. This implies that there will be a discontinuous jump in the cost of compensation contracts when the target effort increases from below the threshold to above. Consider moderate improvements in fundamentals around the threshold level $\left(a_{0}\right)$ that would typically raise the optimal effort level and incentive pay, for example, the agent's effort becomes more productive due to expanded business opportunities. The principal will be reluctant to take advantage of these improvements by adjusting compensation contracts, because enhanced incentives would alter the agent's perception of risk from low to high, and consequently result in a substantial increase in the risk premium. Therefore, compensation contracts exhibit an inertia property, that is, compensation contracts are irresponsive to changes in fundamentals. This feature is consistent with the widespread use of benchmarking (and thus a disconnection between pay and firm performance) in CEO compensation (e.g., Bizjak, Lemmon and Naveen (2008) and Faulkender and Yang (2010)).

Proposition 3. Suppose that the condition (7) holds. Then there exists a cut-off $a_{0}$ such that the cost of contracts will jump discontinuously when the target effort moves from below $a_{0}$ to above $a_{0}$.

\subsection{Relative performance evaluation}

Theories have advocated that any returns that are beyond the agent's control should be isolated from the agent's payoff. This is called relative performance evaluation (RPE). However, empirical studies found that RPE is not frequently used in practice. For example, 
Bertrand and Mullainathan (2001) document that CEOs are rewarded for general market upswings beyond CEO's control, i.e., paid for luck. In this section, we will argue that if the correlation between observable shocks and unobservable shocks is ambiguous, while it is efficient to use RPE for stock compensation, it may not be optimal to use RPE for option compensation.

Specifically, suppose that the shock $\epsilon$ can be divided into two parts: $\epsilon=m+e$, where $m$ represents the part of the shock that is observable and contractible; $e$ is the rest of the shock that is unobservable. $m$ is normally distributed with mean 0 and variance $\sigma_{m}^{2}$. $e$ is normally distributed with mean 0 and variance $\sigma_{e}^{2}$. Let $\rho$ be the correlation between $m$ and $e$. We introduce ambiguity by assuming that $\rho \in[-\bar{\rho}, \bar{\rho}]$, where $0<\bar{\rho}<1 .{ }^{9}$

Since $m$ is observable and contractible (which means that we can use RPE to filter out $m$ from firm value $x$ and use $x-m$ to evaluate the agent's performance), we assume that the principal can use four types of compensation: non-indexed stock compensation (whose payoff has the form $\alpha_{s}+\beta_{s} x$ ), indexed stock compensation (whose payoff has the form $\alpha_{i s}+\beta_{i s}(x-m)$ ), non-indexed option compensation (whose payoff has the form $\alpha_{k}+\beta_{k} \max (x-k, 0)$ ), indexed option compensation (whose payoff has the form $\alpha_{i k}+$ $\left.\beta_{i k} \max (x-m-k, 0)\right)$. Then similar to the argument before, if the agent is granted nonindexed stock compensation, his perception of risk will be the highest possible risk, that is $\sigma_{m}^{2}+\sigma_{e}^{2}+2 \bar{\rho} \sigma_{m} \sigma_{e}$; if the agent is granted indexed stock compensation, his perception of risk will be $\sigma_{e}^{2}$; if the agent is granted non-indexed option compensation and if he is not too risk-averse, his perception of risk can be the lowest possible risk, that is $\sigma_{m}^{2}+\sigma_{e}^{2}-2 \bar{\rho} \sigma_{m} \sigma_{e}$; if the agent is granted indexed option compensation, his perception of risk will be $\sigma_{e}^{2}$. Thus,

\footnotetext{
${ }^{9}$ Thus, the average correlation between $m$ and $e$ is zero.
} 
it is straightforward to show the following lemma.

Lemma 5. If the principal uses stocks to induce effort, using RPE will reduce the cost (i.e., indexed stock is more efficient than non-indexed stock).

If the principal uses options to induce effort, and if the agent is not too risk-averse, using RPE does not always reduce the cost.

Lemma 5 predicts that RPE should be more frequently used for stock compensation than option compensation. Gong, Li and Shin (2010) and Albuquerque (2012) find that RPE is less likely used if firms have more growth opportunities. De Angelis and Grinstein (2011) find that firms rely less on RPE when they are smaller or belong to high-growth industries. For firms with smaller size or more growth opportunities, they usually grant more options to their managers in order to conserve cash while providing significant upside incentives (e.g., Smith and Watts (1992) and Gaver ad Gaver (1993)), and the less frequent use of RPE in these firms is consistent with our model prediction.

The following proposition establishes that non-indexed options can be less costly than indexed stock, if the agent's risk aversion is an intermediate value.

Proposition 4. Let $\sigma_{1}=\sqrt{\sigma_{m}^{2}+\sigma_{e}^{2}-2 \bar{\rho} \sigma_{m} \sigma_{e}}$, and $\sigma_{2}=\sqrt{\sigma_{m}^{2}+\sigma_{e}^{2}+2 \bar{\rho} \sigma_{m} \sigma_{e}}$. Suppose $\sigma_{1}$ is small enough such that $a^{2} \sigma_{e}^{2}>\beta_{k}^{2}\left(\sigma_{1}\right) \sigma_{k}^{2}\left(\sigma_{1}\right)$. If

$$
\frac{2 \beta_{k}\left(\sigma_{1}\right)\left[m_{k}\left(\sigma_{p}\right)-m_{k}\left(\sigma_{1}\right)\right]}{a^{2} \sigma_{e}^{2}-\beta_{k}^{2}\left(\sigma_{1}\right) \sigma_{k}^{2}\left(\sigma_{1}\right)}<\lambda<\min _{\sigma \in\left[\sigma_{1}, \sigma_{2}\right]} \frac{2}{\beta_{k}\left(\sigma_{1}\right)} \frac{m_{k}(\sigma)-m_{k}\left(\sigma_{1}\right)}{\sigma_{k}^{2}(\sigma)-\sigma_{k}^{2}\left(\sigma_{1}\right)}
$$

where $\sigma_{p}$ is the principal's perception of risk for non-indexed options, then non-indexed options are less costly than indexed stock to induce effort. 
From Section 2, we know that indexed stocks are always less costly than indexed options. Thus, if non-indexed options are less costly than indexed stocks, they are also less costly than indexed options. The condition specified in Equation (8) is hence sufficient to imply that using RPE for option compensation is inefficient. This result also provides a rationale for the pay-for-luck puzzle.

Example 2: Similar to Example 1, in Figure 3, we provide a numerical example to show that the condition (8) can be satisfied in a reasonable range of $\lambda$.

In the example, we set $a=0.5, k=0.4, l=0, \sigma_{m}=0.2, \sigma_{e}=0.25$, and $\bar{\rho}=0.9$. Then the possible total firm risk ranges from 0.11 to 0.44 (i.e., $\sigma_{1}=0.11, \sigma_{2}=0.44$ ). From Figure 3, we can see that as the shareholders' perception of risk $\sigma_{p}$ goes from $\sigma_{1}$ to $\sigma_{2}$, the upper bound of $\lambda$ is not affected, and equals to a constant 4.73. This is expected, since the right-hand side of (8) is not affected by $\sigma_{p}$. As $\sigma_{p}$ goes from $\sigma_{1}$ to around 0.24 , the lower bound increases from 0 to 4.73 . This is also consistent with the fact that the left-hand side of (8) increases in $\sigma_{p}$. When $\sigma_{p}$ goes beyond 0.24 , there is no $\lambda$ that can satisfy the condition (8). In sum, as long as $\sigma_{p}$ is not too big (i.e., the principal's valuation of non-indexed options is not too high), there will be a reasonable range of $\lambda$ that can satisfy the condition (8).

\section{Robustness}

In this paper, we restrict the compensation contracts to be stock compensation or option compensation for tractability. In the literature, many papers specify the linear form of contracts for tractability (e.g., Holmstrom and Tirole (1993), Jin (2002), Oyer (2004), 
and Bolton, Scheinkman and Xiong (2006)). In reality, stock and options are the two most widely used instruments in a compensation contract. Since our goal is to compare linear contracts with non-linear contracts in the presence of ambiguity, for the sake of tractability and being consistent with reality, we restrict to stock compensation and option compensation in our model.

So far we have assumed a mean-variance preference for the agent's utility function to get closed-form solutions. The key mechanism behind the results in Section 3 is robust to more general utility functions. The key mechanism behind our results is that if there is ambiguity on firm risk, while stocks always induce the agent to perceive the highest possible risk, options can induce him to perceive the lowest possible risk. The following lemma shows that for any concave utility functions, a linear contract always induces the agent to perceive the highest possible risk.

Lemma 6. If $u(\cdot)$ is a concave function with $u^{\prime}(\cdot)>0$, then for any stock compensation $\alpha_{s}+\beta_{s} x$ with $\beta_{s}>0$ (Recall that $\left.x=a+l+\epsilon\right), E\left[u\left(\alpha_{s}+\beta_{s} x\right)\right]$ is decreasing in $\sigma$.

Lemma 6 implies that for any utility function, a linear contract will induce the (ambiguityaverse) agent to perceive the highest possible risk. Thus, the first half part of our key mechanism survives in a more general setup with any concave utility function. The second half part of the key mechanism states that options can induce the agent to perceive the lowest possible risk. In the following lemma, we will show that it can also carry through in a more general setup.

Lemma 7. If $u(\cdot)$ is a concave function with $u^{\prime}(\cdot)>0$, then for any option compensation $\alpha_{k}+\beta_{k} \max (x-k, 0)$ with $\beta_{k}>0$, there exists a bound $B$ such that if $\left|u^{\prime \prime}(y)\right|<B$ for any 
$y$, then $E\left[u\left(\alpha_{k}+\beta_{k} \max (x-k, 0)\right)\right]$ is increasing in $\sigma$.

Lemma 7 implies that as long as the agent is not too risk-averse, option compensation can induce him to perceive the lowest possible risk. Thus, our results still hold in a setup with a general utility function.

\section{Conclusion}

Standard principal-agent models usually assume that the distribution on the future firm value is known. This paper attempts to study the choice of compensation contracts when there are multiple possible distributions and the agent is averse to ambiguity. The paper finds that when there are multiple possible distributions on firm value, different types of contracts (linear contracts versus non-linear contracts) can induce an ambiguity-averse agent to perceive different distributions, and therefore change the optimality of contracts. In particular, while stocks are always less costly than options in motivating effort in the absence of ambiguity, options can be more efficient than stocks in the presence of ambiguity regarding firm risk. In addition, We find that compensation contracts exhibit an inertia property and benchmark pay is reasonable. When ambiguity regarding the correlation between observable shocks and unobservable shocks exists, CEO pay contingent on the market-wide performances can be optimal. The model also predicts that RPE should be more frequently used for stock compensation than option compensation. 


\section{Appendix}

\section{Proof of Proposition 1}

For stock compensation, the agent's utility is given by $E[u]=\alpha_{s}+\beta_{s}(a+l)-\frac{1}{2} \lambda \beta_{s}^{2} \sigma^{2}-$ $\frac{1}{2} a^{2}$. So to induce the effort $a$, we must have $\beta_{s}=a$. For a fixed target effort $a$, the principal wants to minimize the cost. So $\alpha_{s}$ must be set so that the IR constraint is binding. Thus, it is easy to derive that the cost of the contract (dropping the constant $\frac{1}{2} a^{2}$ ) is: $C_{s}=\frac{1}{2} \lambda a^{2} \sigma^{2}$.

we derive the agent's utility for an option compensation as follows. $E[u]=\alpha_{k}+\beta_{k} m_{k}-$ $\frac{1}{2} \lambda \beta_{k}^{2} \sigma_{k}^{2}-\frac{1}{2} a^{2}$. Taking partial derivative of $m_{k}$ and $\sigma_{k}^{2}$ w.r.t. $a$ yields that

$$
\begin{aligned}
\frac{\partial m_{k}}{\partial a} & =1-\Phi \\
\frac{\partial \sigma_{k}^{2}}{\partial a} & =2 \Phi m_{k}
\end{aligned}
$$

To induce the effort $a$, we must have that $\frac{\partial E[u]}{\partial a} \geq 0$, which yields that

$$
\beta_{k} \geq \frac{(1-\Phi)-\sqrt{\Delta}}{2 \lambda \Phi m_{k}}
$$

where $\Delta=(1-\Phi)^{2}-4 a \lambda \Phi m_{k}$. Similarly, we have the binding IR constraint. So the cost is

$$
C_{k} \geq \frac{1}{2} \lambda\left(\frac{(1-\Phi)-\sqrt{\Delta}}{2 \lambda \Phi m_{k}}\right)^{2} \sigma_{k}^{2}=\frac{2 \lambda a^{2} \sigma^{2} y_{1}}{\left(1+\sqrt{1-4 a \lambda \sigma y_{2}}\right)^{2}}
$$


where

$$
\begin{aligned}
& y_{1}=\frac{\left(1-\Phi-\phi^{2}\right)-\phi(2 \Phi-1) \eta+\Phi(1-\Phi) \eta^{2}}{(1-\Phi)^{2}} \\
& y_{2}=\frac{\Phi[\phi-(1-\Phi) \eta]}{(1-\Phi)^{2}}
\end{aligned}
$$

It can be shown that $y_{1}^{\prime}(\eta) \geq 0$ and $y_{2}^{\prime}(\eta) \geq 0$ (see Feltham and $\mathrm{Wu}(2001)$ ). Thus, $C_{k}$ is increasing in $k$. As $k$ goes to $-\infty, \Phi$ goes to zero. So $\sigma^{2} y_{1}=\frac{\sigma_{k}^{2}}{(1-\Phi)^{2}} \rightarrow \sigma^{2}$, and $\sigma y_{2}=\frac{\Phi m_{k}}{(1-\Phi)^{2}} \rightarrow 0$. Hence, the right-hand side of (9) goes to $\frac{1}{2} \lambda a^{2} \sigma^{2}$ when $k$ goes to $-\infty$. Thus, we obtain that $C_{s}<C_{k}$.

\section{Proof of Lemma 2}

If the agent is granted with stocks, his utility $E[u]=\beta_{s}(a+l)-\frac{1}{2} \lambda \beta_{s}^{2} \sigma^{2}-\frac{1}{2} a^{2}$ is minimized at $\sigma=\sigma_{2}$. So he will always perceive the highest risk $\sigma_{2}$. Following the proof of Proposition 1, we can show that $C_{s}=\frac{1}{2} \lambda a^{2} \sigma_{2}^{2}$.

If the agent is granted with options, his utility is given by

$$
E[u]=\alpha_{k}+\beta_{k}\left(\sigma_{1}\right) m_{k}\left(\sigma_{a}\right)-\frac{1}{2} \lambda \beta_{k}\left(\sigma_{1}\right)^{2} \sigma_{k}\left(\sigma_{a}\right)^{2}-\frac{1}{2} a^{* 2}
$$

let

$$
A_{1}=\left\{\hat{a}: E\left[u(\hat{a}) \mid \sigma_{a}=\sigma_{1}\right]<\min _{\sigma_{a} \in\left(\sigma_{1}, \sigma_{2}\right]} E[u(\hat{a})]\right\}
$$

be the set of effort for which the agent's utility is minimized at the lowest risk $\sigma_{1}$. By condition (5), the target effort $a$ belongs to $A_{1}$, i.e., $a \in A_{1}$. Note that $\beta_{k}\left(\sigma_{1}\right)$ is exactly the number of options to induce the effort $a$ given $\sigma_{a}=\sigma_{1}$. If the agent perceives another 
risk $\sigma \neq \sigma_{1}$ and exerts an effort $\hat{a}$, we must have

$$
E\left[u(\hat{a}) \mid \sigma_{a}=\sigma\right] \leq E\left[u(\hat{a}) \mid \sigma_{a}=\sigma_{1}\right] \leq E\left[u(a) \mid \sigma_{a}=\sigma_{1}\right]
$$

Therefore at the optimum, the agent will perceive the lowest risk $\sigma_{1}$ and exert the target effort $a$. The binding IR constraint yields that $\alpha_{k}+\beta_{k}\left(\sigma_{1}\right) m_{k}\left(\sigma_{1}\right)=\frac{1}{2} \lambda \beta_{k}\left(\sigma_{1}\right)^{2} \sigma_{k}\left(\sigma_{1}\right)^{2}+$ $\frac{1}{2} a^{2}$. From the principal's perspective, the cost (dropping the constant $\frac{1}{2} a^{2}$ ) is $C_{k}=\alpha_{k}+$ $\beta_{k}\left(\sigma_{1}\right) m_{k}\left(\sigma_{p}\right)=\frac{1}{2} \lambda \beta_{k}\left(\sigma_{1}\right)^{2} \sigma_{k}\left(\sigma_{1}\right)^{2}+\beta_{k}\left(\sigma_{1}\right)\left[m_{k}\left(\sigma_{p}\right)-m_{k}\left(\sigma_{1}\right)\right]$.

\section{Proof of Proposition 2}

By Lemma 2, the right hand side of (6) ensures that an option of exercise price $k$ induces the agent to perceive $\sigma_{1}$ and exert the effort $a$. The cost of the contract is $C_{k}=$ $\frac{1}{2} \lambda \beta_{k}\left(\sigma_{1}\right)^{2} \sigma_{k}\left(\sigma_{1}\right)^{2}+\beta_{k}\left(\sigma_{1}\right)\left[m_{k}\left(\sigma_{p}\right)-m_{k}\left(\sigma_{1}\right)\right]$. Since the cost of stock compensation is $C_{s}=$ $\frac{1}{2} \lambda a^{2} \sigma_{2}^{2}$, the left hand side of (6) ensures that the option compensation is less costly.

\section{Analysis of Condition (6)}

We first solve for the right-hand of (6) to obtain an upper bound of $\lambda$. Let $M=$ $\min _{\sigma \in\left[\sigma_{1}, \sigma_{2}\right]} \frac{m_{k}(\sigma)-m_{k}\left(\sigma_{1}\right)}{\sigma_{k}^{2}(\sigma)-\sigma_{k}^{2}\left(\sigma_{1}\right)}$, which does not depend on $\lambda$. From the proof of Proposition 1, we know that $\beta_{k}=\frac{(1-\Phi)-\sqrt{\Delta}}{2 \lambda \Phi m_{k}}$, where $\Delta=(1-\Phi)^{2}-4 a \lambda \Phi m_{k}$. Plugging the formula of $\beta_{k}$ into the right-hand side of (6), we can solve that the right-hand side of (6) is equivalent to $\lambda<\frac{K\left(2-2 \Phi\left(\sigma_{1}\right)-K\right)}{4 a \Phi\left(\sigma_{1}\right) m_{k}\left(\sigma_{1}\right)}$, where $K=4 M \Phi\left(\sigma_{1}\right) m_{k}\left(\sigma_{1}\right)$.

For the left-hand side of (6), it can be simplified to $\lambda \beta_{k}^{2}\left(\sigma_{1}\right) \sigma_{k}^{2}\left(\sigma_{1}\right)+2\left[m_{k}\left(\sigma_{p}\right)-m_{k}\left(\sigma_{1}\right)\right] \beta_{k}\left(\sigma_{1}\right)-$ 
$\lambda a^{* 2} \sigma_{2}^{2}<0$. Note that $\lambda \beta_{k}^{2}=\frac{1-\Phi}{\Phi m_{k}} \beta_{k}-\frac{a}{\Phi m_{k}}$, so the left-hand side of (6) is equivalent to

$$
\left[\frac{\left(1-\Phi\left(\sigma_{1}\right)\right) \sigma_{k}^{2}\left(\sigma_{1}\right)}{\Phi\left(\sigma_{1}\right) m_{k}\left(\sigma_{1}\right)}+2\left[m_{k}\left(\sigma_{p}\right)-m_{k}\left(\sigma_{1}\right)\right]\right] \beta_{k}\left(\sigma_{1}\right)-\frac{a \sigma_{k}^{2}\left(\sigma_{1}\right)}{\Phi\left(\sigma_{1}\right) m_{k}\left(\sigma_{1}\right)}-\lambda a^{2} \sigma_{2}^{2}<0
$$

It is easy to check that the left-hand side of (10) is convex in $\lambda$, and is non-negative when $\lambda=0$. So there could be three cases:

Case 1: the left-hand side of (10) never crosses with the x-axis, which means that (10) will never be satisfied. In this case, the condition (6) cannot be satisfied.

Case 2: the left-hand side of (10) crosses with the x-axis once. Let $\lambda_{1}$ denote the crossed point, then the condition (6) is equivalent to $\lambda_{1}<\lambda<\frac{K\left(2-2 \Phi\left(\sigma_{1}\right)-K\right)}{4 a \Phi\left(\sigma_{1}\right) m_{k}\left(\sigma_{1}\right)}$.

Case 3: the left-hand side of (10) crosses with the x-axis twice. Let $\lambda_{1}<\lambda_{2}$ denote the two crossed points, then the condition (6) is equivalent to $\lambda_{1}<\lambda<\min \left(\frac{K\left(2-2 \Phi\left(\sigma_{1}\right)-K\right)}{4 a \Phi\left(\sigma_{1}\right) m_{k}\left(\sigma_{1}\right)}, \lambda_{2}\right)$.

\section{Proof of Lemma 3}

Suppose the principal uses a contract $w=\alpha+\beta_{s} x+\beta_{k} \max (x-k, 0)$ to induce the agent to perceive $\sigma_{1}$ and exert the target effort $a_{2}$. Then we must have that for any $\sigma_{a} \in\left(\sigma_{1}, \sigma_{2}\right]$,

$$
\begin{aligned}
& \alpha+\beta_{s}\left(a_{2}+l\right)+\beta_{k} m_{k}\left(\sigma_{1}\right)-\frac{1}{2} \lambda\left[\beta_{s}^{2} \sigma_{1}^{2}+\beta_{k}^{2} \sigma_{k}\left(\sigma_{1}\right)^{2}+2 \beta_{s} \beta_{k} \sigma_{0, k}\left(\sigma_{1}\right)\right] \\
\leq & \alpha+\beta_{s}\left(a_{2}+l\right)+\beta_{k} m_{k}\left(\sigma_{a}\right)-\frac{1}{2} \lambda\left[\beta_{s}^{2} \sigma_{a}^{2}+\beta_{k}^{2} \sigma_{k}\left(\sigma_{a}\right)^{2}+2 \beta_{s} \beta_{k} \sigma_{0, k}\left(\sigma_{a}\right)\right],
\end{aligned}
$$

where $\sigma_{0, k}$ denotes the covariance between stocks and options. The inequality can be simplified to

$\frac{1}{2} \lambda\left[\frac{\beta_{s}^{2}}{\beta_{k}}\left(\sigma_{a}^{2}-\sigma_{1}^{2}\right)+\beta_{k}\left(\sigma_{k}\left(\sigma_{a}\right)^{2}-\sigma_{k}\left(\sigma_{1}\right)^{2}\right)+2 \beta_{s}\left(\sigma_{0, k}\left(\sigma_{a}\right)-\sigma_{0, k}\left(\sigma_{1}\right)\right)\right] \leq m_{k}\left(\sigma_{a}\right)-m_{k}\left(\sigma_{1}\right)$. 
Note that $\sigma_{k}(\sigma)^{2}, \sigma_{0, k}(\sigma)$ and $m_{k}(\sigma)$ are increasing in $\sigma$. As we gradually reduce $\beta_{s}$ and $\beta_{k}$, the inequality above can still hold, so the agent will continue to perceive the lowest risk $\sigma_{1}$. We keep reducing $\beta_{s}$ and $\beta_{k}$ until $a_{1}$ is induced. Then we are done.

\section{Proof of Lemma 4}

When the effort $a$ is induced, the agent's utility is

$$
E\left[u \mid \sigma_{a}\right]=\alpha+\beta_{s}(a+l)+\beta_{k} m_{k}\left(\sigma_{a}\right)-\frac{1}{2} \lambda\left[\beta_{s}^{2} \sigma_{a}^{2}+\beta_{k}^{2} \sigma_{k}\left(\sigma_{a}\right)^{2}+2 \beta_{s} \beta_{k} \sigma_{0, k}\left(\sigma_{a}\right)\right]-\frac{1}{2} a^{2} .
$$

We can calculate that $\frac{\partial^{2} m_{k}(\sigma)}{\partial \sigma^{2}}=\frac{\phi \eta^{2}}{\sigma} ; \frac{\partial^{2} \sigma_{k}(\sigma)^{2}}{\partial \sigma^{2}}=2\left(1-\Phi-\phi^{2}+\phi \eta-\phi^{2} \eta^{2}+\phi \eta^{3}-\phi \Phi \eta^{3}\right)$; $\frac{\partial^{2} \sigma_{0, k}(\sigma)}{\partial \sigma^{2}}=2(1-\Phi)+2 \phi \eta+\phi \eta^{3}$.

Since $a$ is induced, we must have

$$
\max _{\sigma \in\left[\sigma_{1}, \sigma_{2}\right]} \frac{\partial E[u \mid \sigma]}{\partial a} \geq 0
$$

Let $\sigma_{0} \in \arg \max _{\sigma \in\left[\sigma_{1}, \sigma_{2}\right]} \frac{\partial E[u \mid \sigma]}{\partial a}$. Then we obtain that

$$
\beta_{s}+\beta_{k}\left(1-\Phi\left(\sigma_{0}\right)\right)-\frac{1}{2} \lambda\left[\beta_{k}^{2} \frac{\partial \sigma_{k}\left(\sigma_{0}\right)^{2}}{\partial a}+2 \beta_{s} \beta_{k} \frac{\partial \sigma_{0, k}\left(\sigma_{0}\right)}{\partial a}\right]-a \geq 0
$$

Since $\frac{\partial \sigma_{k}\left(\sigma_{0}\right)^{2}}{\partial a}>0$ and $\frac{\partial \sigma_{0, k}\left(\sigma_{0}\right)}{\partial a}>0, \beta_{s}+\beta_{k}>a$. 
Taking the second-order derivative of $E\left[u \mid \sigma_{a}\right]$ w.r.t. $\sigma_{a}$ yields that

$$
\begin{aligned}
\frac{\partial^{2} E[u]}{\partial \sigma_{a}^{2}} & =\beta_{k} \frac{\phi \eta^{2}}{\sigma_{a}}-\frac{1}{2} \lambda\left[2 \beta_{s}^{2}\right. \\
& \left.+2 \beta_{k}^{2}\left(1-\Phi-\phi^{2}+\phi \eta-\phi^{2} \eta^{2}+\phi \eta^{3}-\phi \Phi \eta^{3}\right)+2 \beta_{s} \beta_{k}\left(2(1-\Phi)+2 \phi \eta+\phi \eta^{3}\right)\right] \\
& <\beta_{k}\left[\frac{\phi \eta^{2}}{\sigma_{a}}-\frac{1}{2} \lambda\left[2 \beta_{k}\left(1-\Phi-\phi^{2}+\phi \eta-\phi^{2} \eta^{2}+\phi \eta^{3}-\phi \Phi \eta^{3}\right)+2 \beta_{s}\left(2(1-\Phi)+2 \phi \eta+\phi \eta^{3}\right)\right]\right]
\end{aligned}
$$

If $|k-a-l|<\sigma_{1} \cdot \min \left(1, \sqrt{\frac{1}{2} \lambda a \sigma_{1}}\right)$, then $\left|\eta\left(\sigma_{a}\right)\right|<\min \left(1, \sqrt{\frac{1}{2} \lambda a \sigma_{1}}\right)$. Thus, $\frac{\phi \eta^{2}}{\sigma_{a}}<$ $\frac{\phi \cdot \frac{1}{2} \lambda a \sigma_{1}}{\sigma_{1}}=\frac{1}{2} \lambda a \phi$. Using Matlab, we can draw the graph for the functions $1-\Phi-\phi^{2}+\phi \eta-$ $\phi^{2} \eta^{2}+\phi \eta^{3}-\phi \Phi \eta^{3}$ and $2(1-\Phi)+2 \phi \eta+\phi \eta^{3}$ with $|\eta|<1$. From Figure 4 , we can see that both functions are greater than 0.5 when $|\eta|<1$. Note that $\phi<\frac{1}{\sqrt{2 \pi}}$ for any $\eta$. Thus,

$$
\frac{\partial^{2} E[u]}{\partial \sigma_{a}^{2}}<\frac{1}{2} \lambda \beta_{k}\left[\frac{1}{\sqrt{2 \pi}} a-\left(\beta_{s}+\beta_{k}\right)\right]<0 .
$$

Thus, we prove that if $|k-a-l|<\sigma_{1} \cdot \min \left(1, \sqrt{\frac{1}{2} \lambda a \sigma_{1}}\right), \frac{\partial^{2} E[u]}{\partial \sigma_{a}^{2}}<0$. Since $E\left[u \mid \sigma_{a}\right]$ is concave in $\sigma_{a}, E\left[u \mid \sigma_{a}\right]$ must be minimized at $\sigma_{a}=\sigma_{1}$ or $\sigma_{a}=\sigma_{2}$. So the agent will perceive either the lowest risk $\sigma_{1}$ or the highest risk $\sigma_{2}$.

\section{Proof of Proposition 4}

It is straightforward from the proofs of Lemma 2 and Proposition 2.

\section{Proof of Lemma 6}


We can calculate that

$$
\begin{aligned}
E\left[u\left(\alpha_{s}+\beta_{s} x\right)\right] & =\int_{-\infty}^{\infty} u\left(\alpha_{s}+\beta_{s}(a+l+y)\right) \frac{1}{\sigma \sqrt{2 \pi}} e^{-\frac{y^{2}}{2 \sigma^{2}}} d y, \text { let } z=\frac{y}{\sigma} \\
& =\int_{-\infty}^{\infty} u\left(\alpha_{s}+\beta_{s}(a+l+z \sigma)\right) \frac{1}{\sqrt{2 \pi}} e^{-\frac{z^{2}}{2}} d z .
\end{aligned}
$$

Thus, taking the derivative w.r.t. $\sigma$ yields that

$$
\begin{aligned}
& \frac{\partial}{\partial \sigma} E\left[u\left(\alpha_{s}+\beta_{s} x\right)\right] \\
= & \int_{-\infty}^{\infty} u^{\prime}\left(\alpha_{s}+\beta_{s}(a+l+z \sigma)\right) \beta_{s} z \frac{1}{\sqrt{2 \pi}} e^{-\frac{z^{2}}{2}} d z, \\
= & \int_{0}^{\infty} u^{\prime}\left(\alpha_{s}+\beta_{s}(a+l+z \sigma)\right) \beta_{s} z \frac{1}{\sqrt{2 \pi}} e^{-\frac{z^{2}}{2}} d z-\int_{0}^{\infty} u^{\prime}\left(\alpha_{s}+\beta_{s}(a+l-z \sigma)\right) \beta_{s} z \frac{1}{\sqrt{2 \pi}} e^{-\frac{z^{2}}{2}} d z .
\end{aligned}
$$

Since $u$ is concave, it means that $u^{\prime}(\cdot)$ is a decreasing function. So $\int_{0}^{\infty} u^{\prime}\left(\alpha_{s}+\beta_{s}(a+\right.$ $l+z \sigma)) \beta_{s} z \frac{1}{\sqrt{2 \pi}} e^{-\frac{z^{2}}{2}} d z<\int_{0}^{\infty} u^{\prime}\left(\alpha_{s}+\beta_{s}(a+l)\right) \beta_{s} z \frac{1}{\sqrt{2 \pi}} e^{-\frac{z^{2}}{2}} d z$, and $\int_{0}^{\infty} u^{\prime}\left(\alpha_{s}+\beta_{s}(a+l-\right.$ $z \sigma)) \beta_{s} z \frac{1}{\sqrt{2 \pi}} e^{-\frac{z^{2}}{2}} d z>\int_{0}^{\infty} u^{\prime}\left(\alpha_{s}+\beta_{s}(a+l)\right) \beta_{s} z \frac{1}{\sqrt{2 \pi}} e^{-\frac{z^{2}}{2}} d z$. Thus, $\frac{\partial}{\partial \sigma} E\left[u\left(\alpha_{s}+\beta_{s} x\right)\right]<0$.

Hence, $E\left[u\left(\alpha_{s}+\beta_{s} x\right)\right]$ is decreasing in $\sigma$.

\section{Proof of Lemma 7}

Similar to the proof of Lemma 6 , we can calculate that

$$
\begin{aligned}
& E\left[u\left(\alpha_{k}+\beta_{k} \max (x-k, 0)\right)\right] \\
= & \int_{-\infty}^{\infty} u\left(\alpha_{k}+\beta_{k} \max (a+l+\sigma z-k, 0)\right) \frac{1}{\sigma \sqrt{2 \pi}} e^{-\frac{z^{2}}{2}} d z, \\
= & u\left(\alpha_{k}\right) \Phi\left(\frac{k-a-l}{\sigma}\right)+\int_{\frac{k-a-l}{\sigma}}^{\infty} u\left(\alpha_{k}+\beta_{k}(a+l+z \sigma-k)\right) \frac{1}{\sqrt{2 \pi}} e^{-\frac{z^{2}}{2}} d z .
\end{aligned}
$$


Taking the derivative w.r.t. $\sigma$ yields that

$$
\frac{\partial}{\partial \sigma} E\left[u\left(\alpha_{k}+\beta_{k} \max (x-k, 0)\right)\right]=\int_{\frac{k-a-l}{\sigma}}^{\infty} u^{\prime}\left(\alpha_{k}+\beta_{k}(a+l+z \sigma-k)\right) \beta_{k} z \frac{1}{\sqrt{2 \pi}} e^{-\frac{z^{2}}{2}} d z
$$

If $k-a-l \geq 0$, then it is obvious that $\frac{\partial}{\partial \sigma} E\left[u\left(\alpha_{k}+\beta_{k} \max (x-k, 0)\right)\right]>0$. If $k-a-l<0$, then since $\int_{\frac{k-a-l}{\sigma}}^{\infty} u^{\prime}\left(\alpha_{k}+\beta_{k}(a+l+z \sigma-k)\right) \beta_{k} z \frac{1}{\sqrt{2 \pi}} e^{-\frac{z^{2}}{2}} d z=\int_{\frac{k-a-l}{\sigma}}^{\frac{a+l-k}{\sigma}} u^{\prime}\left(\alpha_{k}+\beta_{k}(a+l+\right.$ $z \sigma-k)) \beta_{k} z \frac{1}{\sqrt{2 \pi}} e^{-\frac{z^{2}}{2}} d z+\int_{\frac{a+l-k}{\sigma}}^{\infty} u^{\prime}\left(\alpha_{k}+\beta_{k}(a+l+z \sigma-k)\right) \beta_{k} z \frac{1}{\sqrt{2 \pi}} e^{-\frac{z^{2}}{2}} d z$. The first part equals to zero when $u^{\prime \prime}(\cdot)=0$, and the second part is always positive, so there must exist a bound $B$ such that if $\left|u^{\prime \prime}(y)\right|<B$ for any $y$, then $\frac{\partial}{\partial \sigma} E\left[u\left(\alpha_{k}+\beta_{k} \max (x-k, 0)\right)\right]>0$, i.e., $E\left[u\left(\alpha_{k}+\beta_{k} \max (x-k, 0)\right)\right]$ is increasing in $\sigma$. 


\section{References}

[1] Ahn, David, Syngjoo Choi, Douglas Gale and Shachar Kariv, 2009, "Estimating Ambiguity-Aversion in a Portfolio Choice Experiment," Working Paper.

[2] Albuquerque, Ana, 2012, "Do Growth-Option Firms Use Less Relative Performance Evaluation," Working Paper.

[3] Bertrand, Marianne and Sendhil Mullainathan, 2001, "Are CEOs Rewarded for Luck? The Ones Without Principals Are," Quarterly Journal of Economics, 116, 901-932.

[4] Bettis, J. Carr, John M. Bizjak and Michael L. Lemmon, 2005, "Exercise Behavior, Valuation, and the Incentives Effects of Employee Stock Options," Journal of Financial Economics 76, 445-470.

[5] Bizjak, John M., Michael Lemmon and Lalitha Naveen, 2008, "Does the Use Peer Groups Contribute to Higher Pay and Less Efficient Compensation," Journal of Financial Economics 90, 152-168.

[6] Bolton, Patrick, José Scheinkman and Wei Xiong, 2006, "Executive Compensation and Short-Termist Behaviour in Speculative Markets," Review of Economic Studies 73, 577-610.

[7] Carpenter, Jennifer, 2000, "Does Option Compensation Increase Managerial Risk Appetite," Journal of Finance 55, 2311-2331.

[8] Carroll, Gabriel, 2015, "Robustness and Linear Contracts," American Economic Review 105(2), 536-563. 
[9] De Angelis, David and Yaniv Grinstein, 2011, "Relative Performance Evaluation in CEO Compensation: Evidence from the 2006 Disclosure Rules," Working Paper.

[10] Dickhaut, John, Timothy Shields, and Jack Stecher, 2011, "Generating Ambiguity in the Laboratory," Management Science 57(4), 705-712.

[11] Dittmann, Ingolf and Ernst Maug, 2007, "Lower Salaries and No Options? On the Optimal Structure of Executive Pay, " Journal of Finance 62, 303-343.

[12] Dittmann, Ingolf, Ernst Maug and Oliver Spalt, 2010, "Sticks or Carrots? Optimal CEO Compensation When Managers Are Loss-Averse, " Journal of Finance, forthcoming.

[13] Dittmann, Ingolf and Ko-Chia Yu, 2011, "How Important Are Risk-Taking Incentives in Executive Compensation?" Working Paper.

[14] Easley, David and Maureen O'Hara, 2009, "Ambiguity and Nonparticipation: The Role Regulation," Review of Financial Studies 22, 1818-1843.

[15] Easley, David and Maureen O'Hara, 2010, "Liquidity and Valuation in an Uncertain World," Journal of Financial Economics 97, 1-11.

[16] Edmans, Alex and Xavier Gabaix, 2011, "Tractability in Incentive Contracting," Review of Financial Studies 24, 2865-2894.

[17] Ellsberg, D., 1961, "Risk, Ambiguity, and the Savage Axioms," Quarterly Journal of Economics 75, 643-669. 
[18] Epstein, Larry G. and Tan Wang, 1994, "Intertemporal Asset Pricing under Knightian Uncertainty," Econometrica 62, 283-322.

[19] Epstein, Larry G. and Martin Schneider, 2008, “Ambiguity, Information Quality, and Asset Pricing," Journal of Finance 63, 197-228.

[20] Faulkender, Michael and Jun Yang, 2010, "Inside the Black Box: The Role and Composition of Compensation Peer Groups," Journal of Financial Economics 96, 257-270.

[21] Feltham, Gerald A. and Martin G. H. Wu, 2001, "Incentive Efficiency of Stock versus Options," Review of Accounting Studies 6, 7-28.

[22] Gilboa, Itzchak and David Schmeidler, 1989, "Maxmin Expected Utility with Nonunique Prior," Journal of Mathematical Economics 18, 141-153.

[23] Gong, Guojin, Laura Yue Li and Jae Yong Shin, 2010, "Relative Performance Evaluation and Related Peer Groups in Executive Compensation Contracts," Working Paper.

[24] Grossman, Sanford and Oliver Hart, 1983, "An Analysis of the Principal-Agent Problem," Econometrica 51, 7-45.

[25] Hayes, Rachel M., Michael Lemmon and Mingming Qiu, 2010, "Stock Options and Managerial Incentives for Risk-Taking: Evidence from FAS 123R," Working Paper.

[26] He, Zhiguo, Si Li, Bin Wei and Jianfeng Yu, 2014, "Uncertainty, Risk, and Incentives: Theory and Evidence," Management Science 60, 206-226. 
[27] He, Zhiguo, Bin Wei, and Jianfeng Yu, 2014, "Optimal Long-term Contracting with Learning," Working Paper.

[28] Holmstrom, Bengt and Paul Milgrom, 1987, "Aggregation and Linearity in the Provision of Intertemporal Incentives," Econometrica 55, 303-328.

[29] Holmstrom, Bengt and Jean Tirole, 1993, "Market Liquidity and Performance Monitoring," Journal of Political Economy 101, 678-709.

[30] Illeditsch, Philipp, 2011, "Ambiguous Information, Portfolio Inertia, and Excess Volatility," Journal of Finance, forthcoming.

[31] Jin, Li, 2002, "CEO Compensation, Diversification, and Incentives," Journal of Financial Economics 66, 29-63.

[32] Karni, Edi, 2009, "A Reformulation of the Maxmin Expected Utility Model with Application to Agency Theory," Journal of Mathematical Economics 45, 97-112.

[33] Kellner, Christian, 2015, "Tournaments as Response to Ambiguity Aversion in Incentive Contracts," Journal of Economic Theory, 159, 627-655.

[34] Kellner, Christian and Gerhard Riener, 2012, "The Effect of Ambiguity Aversion on Reward Scheme Choice," Working Paper.

[35] Mukerji, Sujoy, 2010, "Ambiguity Aversion and Cost-Plus Procurement Contracts," Working Paper.

[36] Murphy, Kevin, 1999, "Executive Compensation ," in O. Ashenfelter and D. Card (eds), Handbook of Labor Economics (New York: Elsevier/North-Holland). 
[37] Oyer, Paul, 2004, "Why Do Firms Use Incentives That Have No Incentive Effects," Journal of Finance 59, 1619-1650.

[38] Prendergast, Canice, 2002, "The Tenuous Trade-Off between Risk and Incentives," Journal of Political Economy, 110, 1071-1102.

[39] Ross, Stephen, 2004, "Incentives and the Duality of Risk Aversion and Riskiness," Journal of Finance 59, 207-225.

[40] Smith, Clifford and Ross Watts, 1992, "The Investment Opportunity Set and Corporate Financing, Dividend, and Compensation Policies," Journal of Financial Economics, 32, 263-292.

[41] Sung Jaeyoung, 1995, "Linearity with Project Selection and Controllable Diffusion Rate in Continuous-Time Principal-Agent Problems," The RAND Journal of Economics 26, 720-743.

[42] Viero, Marie-Louise, 2012, "Contracting in Vague Environments, " textitAmerican Economic Journal: Microeconomics, 4(2), 104C130.

[43] Weinschenk, Philipp, 2010, "Moral Hazard and Ambiguity," Working Paper. 
Figure 1: Market Value and Risk Premium
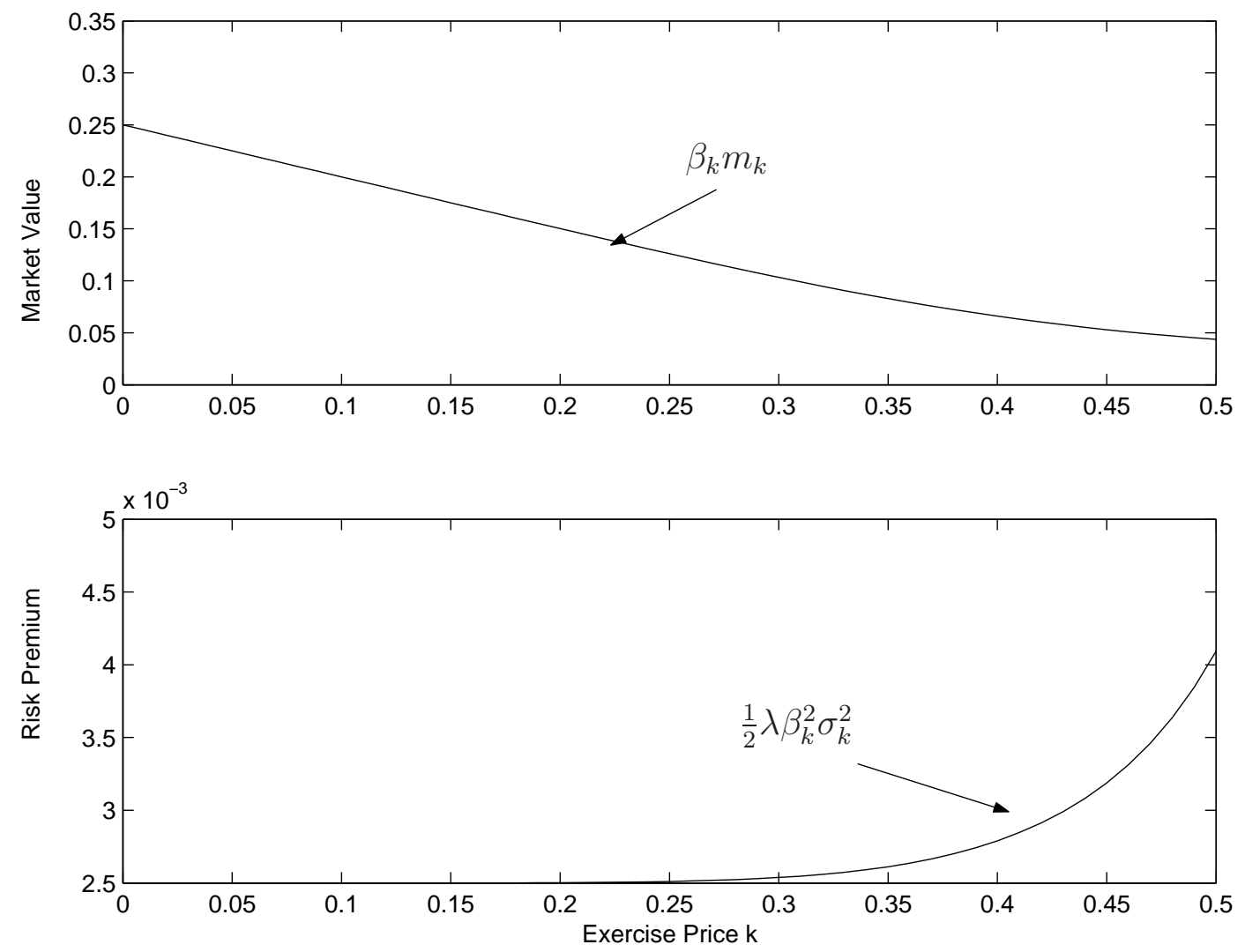

This picture describes a numerical example showing that how the market value and risk premium of an option change with respect to the change in the exercise price $k$. In the example, we set $a=0.5, l=0, \sigma=0.1$, and $\lambda=2$. 
Figure 2: The relation between $\sigma_{p}$ and lower and upper bound of $\lambda$

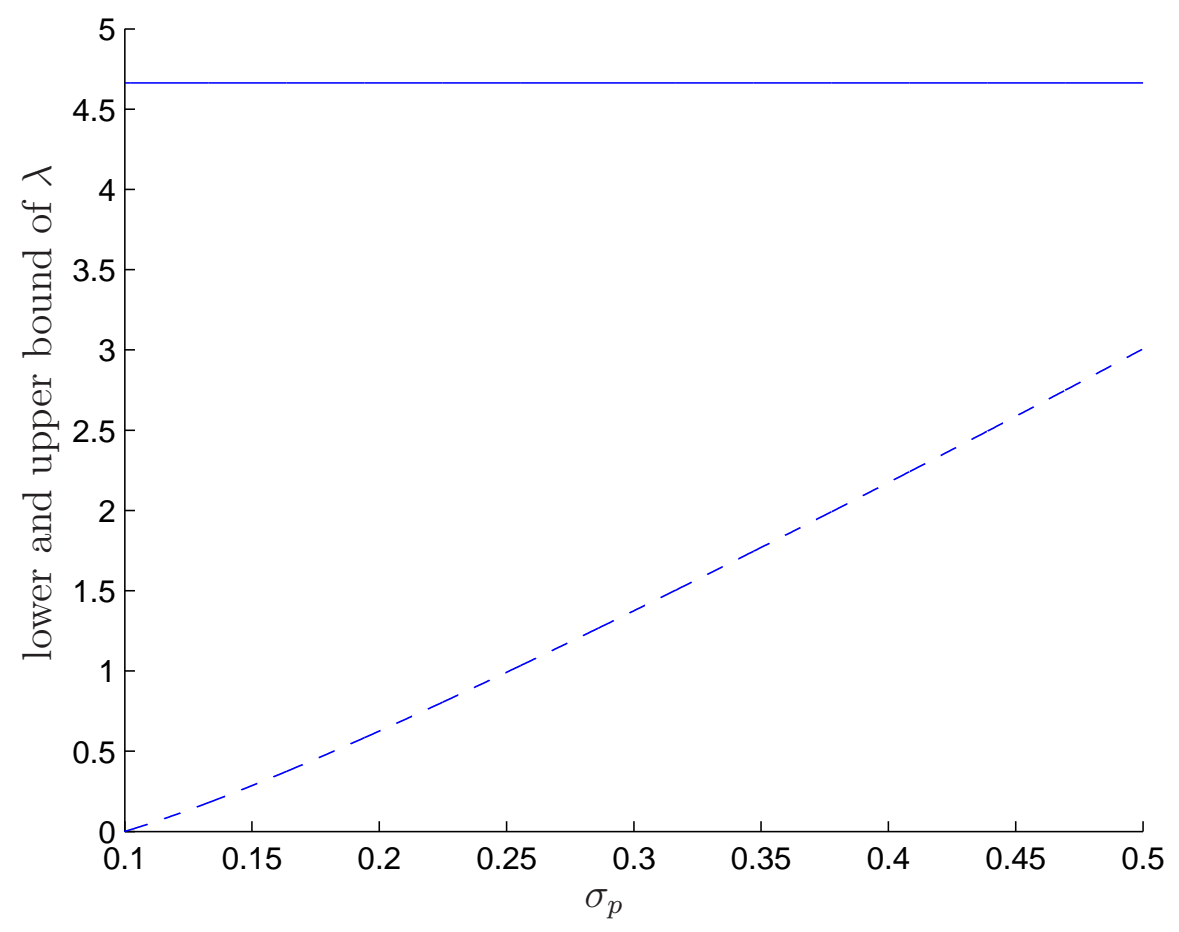

In the example, we set $a=0.5, k=0.4, l=0, \sigma_{1}=0.1$, and $\sigma_{2}=0.5$. The dotted line represents the lower bound of $\lambda$ (with respect to the change of $\sigma_{p}$ ). The solid line represents the upper bound of $\lambda$ (with respect to the change of $\sigma_{p}$ ). 
Figure 3: The relation between $\sigma_{p}$ and lower and upper bound of $\lambda$

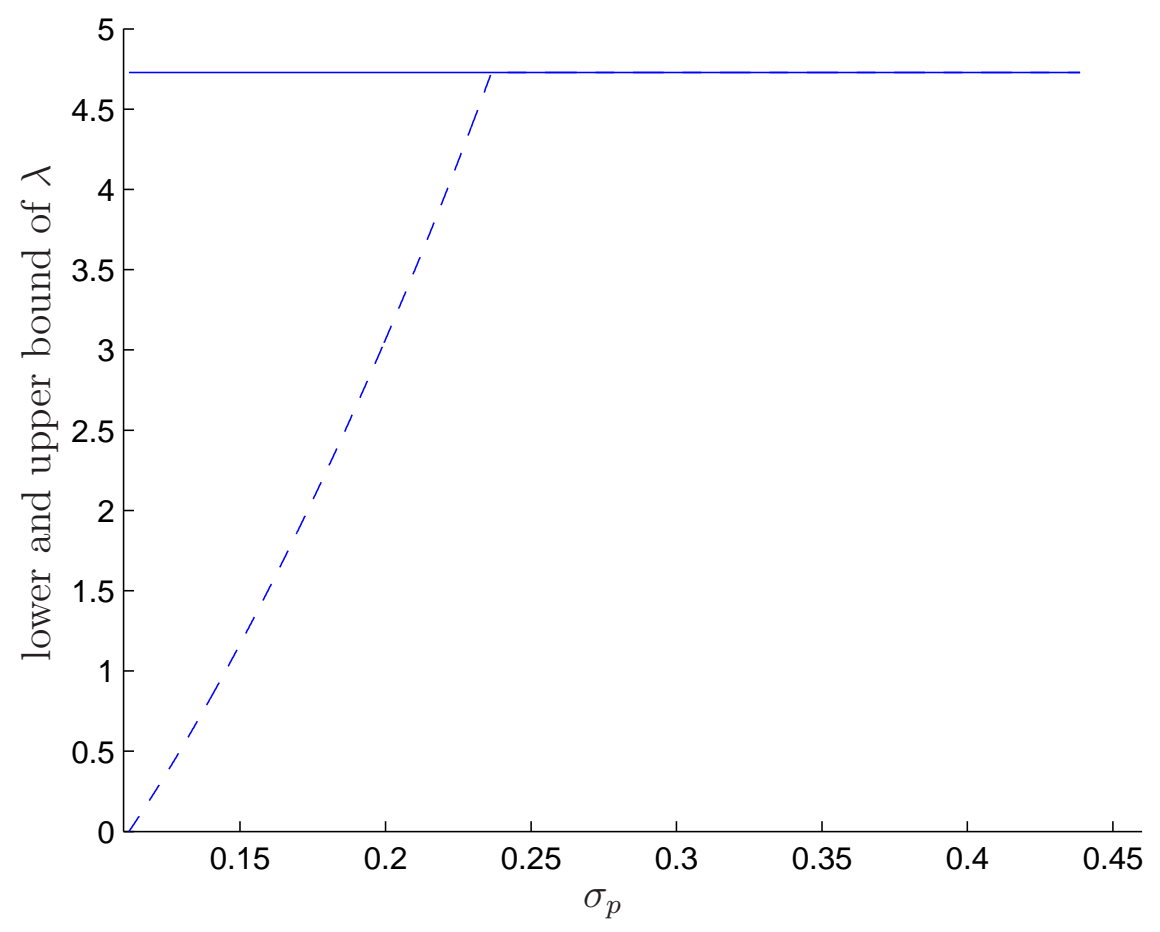

In the example, we set $a=0.5, k=0.4, l=0, \sigma_{m}=0.2, \sigma_{e}=0.25$, and $\bar{\rho}=0.9$. The dotted line represents the lower bound of $\lambda$ (with respect to the change of $\sigma_{p}$ ). The solid line represents the upper bound of $\lambda$ (with respect to the change of $\sigma_{p}$ ). 
Figure 4: The two functions

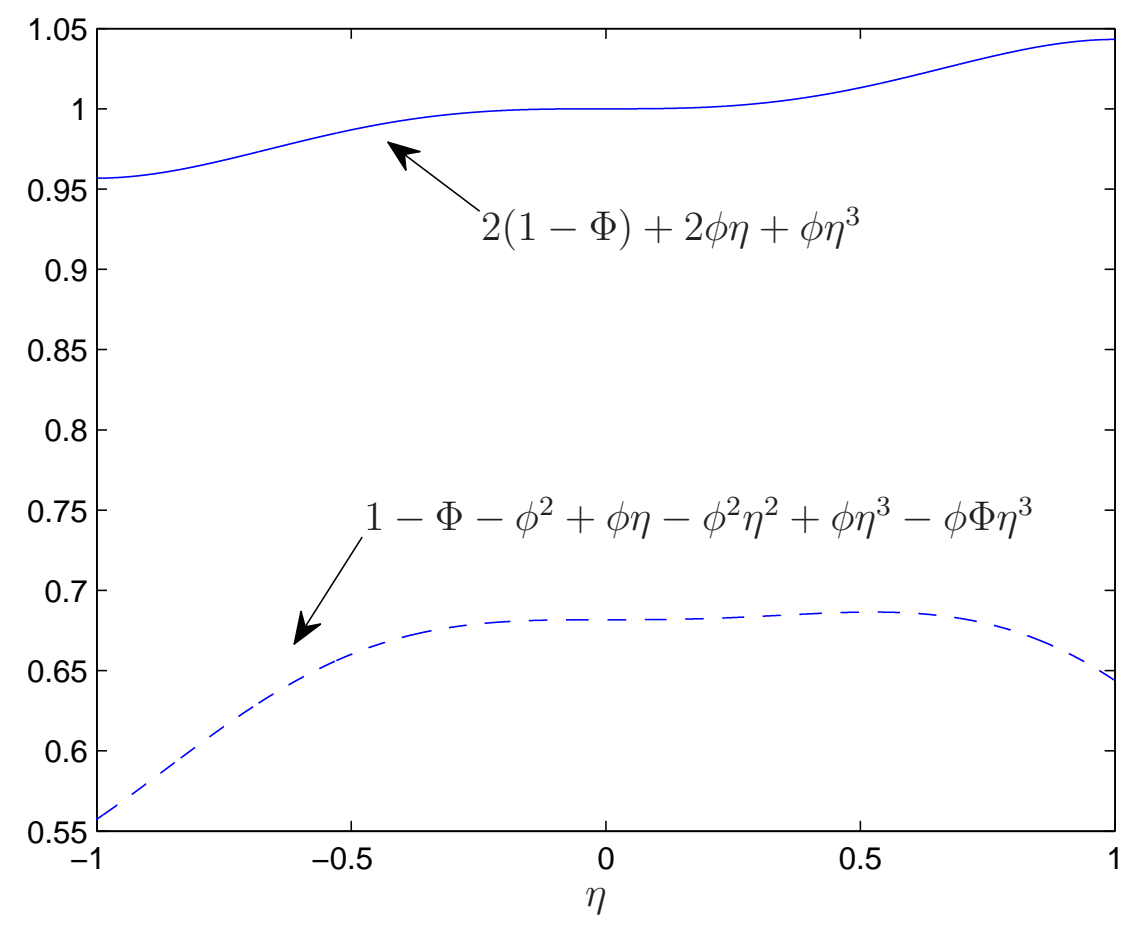

